experienced hands robotic IDS proved feasible in cases with a pelvic mass up to $8 \mathrm{~cm}$. Robotic surgery is not suitable for peritoneal disease covering the anterior abdominal wall close to port sites but does facilitate pelvic and diaphragmatic stripping and arguably provides better visualisation of these peritoneal surfaces in women with high BMI. The planned multicentre MIRRORS-RCT will assess whether robotic IDS offers improved quality of life and recovery with non-inferior progression-free and overall survival. We present the evolution of our surgical technique with illustrative surgical videos and qualitative patient feedback, supported by the objective surgical outcomes for this trial.

Trial Registration

ClinicalTrials gov: NCT04402333 (https://clinicaltrials.gov/ct2/ show/study/NCT04402333)

ISRCTN ISRCTN74222598 (https://www.isrctn.com/

ISRCTN74222598)

\section{LAPAROSCOPIC SALVAGE LYMPHADENECTOMY IN ISOLATED LYMPH NODE RECURRENT OVARIAN CANCER PATIENTS}

${ }^{1,2} \mathrm{C}$ Ronsini* ${ }^{2}{ }^{2} \mathrm{~A}$ Foresta, ${ }^{2} \mathrm{R}$ Oliva, ${ }^{2} \mathrm{~V}$ Gallitelli, ${ }^{2} \mathrm{C}$ Certelli, ${ }^{1} \mathrm{~A}$ Rosati, ${ }^{1,2} \mathrm{C}$ Conte, 1,2 2 Cappuccio, ${ }^{1} V$ Gallotta, ${ }^{1,2} \mathrm{~A}$ Fagotti, ${ }^{1,2} \mathrm{G}$ Scambia. ${ }^{1}$ Woman, Child and Public Health Department, Fondazione Policlinico Universitario A. Gemelli IRCCS, Roma, Italy; ${ }^{2}$ Università Cattolica del Sacro Cuore Sede di Roma, Roma, Italy

\subsection{6/ijgc-2021-ESG0.515}

Introduction/Background* Isolated lymph node recurrence (ILNR) in selected platinum-sensitive recurrent ovarian cancer (psROC) patients is a very infrequent event (12\% to $37 \%$ ) and represent a less aggressive and indolent pattern of ROC. ILNR best treatment is represented by secondary cytoreductive surgery (SCS) with laparotomic or laparoscopic approach, given its non-chemo-sensivity, in particular when occurs in psROC patients in a number of metastatic lymph-nodes $\leq 3$. ILNR favourable prognosis is certified by a median postrelapse survival which is around 37 months and without a certain association with BRCA mutational status. The objective of this video-article is to show the laparoscopic treatment for ILNR.

Methodology

Result(s)* A case of 68 years-old patient affected by psROC is reported. The woman previously underwent first line platinum-based chemotherapy and interval debulking surgery, followed by bevacizumab maintenance therapy. Follow-up CT scan revealed isolated lymphadenopathy in the left iliac-obturatory region. The video shows a laparoscopic salvage lymphadenectomy, with complete pelvic and aortic dissection. Obviously, the surgeon should be able to prevent and to manage severe vascular complications. It is mandatory to recognize any anatomical anomalies and expose the surgical field to prevent and repair retroperitoneal injuries. In this sense, a preoperative radiological workup is necessary to better localize the lymph node disease. The patient was discharged in the $2^{\text {nd }}$ post-operative day without any intra/post-operative complication. The final histology revealed ROC in $1 / 19$ pelvic and aortic nodes.

Conclusion* Salvage lymphadenectomy for ILNR represent a challenging situation and an accurate preoperative study in different clinical situations is essential according to the PFI, number and site of metastases and to the BRCA mutational status. In selected cases, lymphadenectomy performed in a referral centre for gynaecological oncology, with a minimally invasive laparoscopic approach, is a reliable surgical option and could represent a very good alternative to laparotomy.

\section{NEOADJUVANT CHEMOTHERAPY VERSUS PRIMARY DEBULKING SURGERY IN FIGO STAGE III AND IV EPITHELIAL OVARIAN, TUBAL OR PERITONEAL CANCER}

${ }^{1} \mathrm{~A}$ Tzanis ${ }^{*},{ }^{2} \mathrm{CR}$ lavazzo, ${ }^{3} \mathrm{~A}$ Hadjivasilis, ${ }^{4} \mathrm{H}$ Tsouvali, ${ }^{5} \mathrm{G}$ Antoniou, ${ }^{6} \mathrm{~S}$ Antoniou. ${ }^{1}$ University of Thessaly, Medicine, Larissa, Greece; ${ }^{2}$ Metaxa Cancer Hospital of Piraeus, Gynaecologic Oncology, Piraeus, Greece; ${ }^{3}$ Agios Savvas Cancer Hospital, Medical Oncology, Athens, Greece; ${ }^{4}$ University Hospital of loannina, loannina, Greece; ${ }^{5}$ Manchester University NHS Foundation Trust, Manchester, UK; ${ }^{6}$ Mediterranean Hospital of Cyprus, Limassol, Cyprus

\subsection{6/ijgc-2021-ESGO.516}

Introduction/Background* The standard of treatment for advanced epithelial ovarian cancer (EOC) is primary debulking surgery (PDS) followed by platinum-based systemic chemotherapy. Due to the presence of extensive metastatic disease in most of the cases, primary debulking surgery can be an aggressive procedure associated with high peri-operative morbidity and mortality. In this study we aim to investigate whether neoadjuvant chemotherapy (NACT) offers superior survival rates, less peri-operative morbidity and mortality and better quality of life compared to primary debulking surgery in patients with advanced epithelial ovarian cancer.

Methodology We searched the electronic databases PubMed, Cochrane Central Register of Controlled trials, and Scopus from inception to March 2021. We considered randomised controlled trials (RCTs) comparing NACT with PDS for women with EOC stages III and IV. The primary outcomes were overall survival and progression-free survival. Secondary outcomes were optimal cytoreduction rates, peri-operative adverse events, and quality of life.

Result(s)* Six RCTs with a total of 1901 participants were included. Meta-analysis demonstrated similar overall survival $(\mathrm{HR}=0.96,95 \%$ CI $[0.86-1.07])$ and progression-free survival

$(\mathrm{HR}=0.98,95 \%$ CI $[0.89-1.08])$ between NACT and PDS. Subgroup analyses did not demonstrate higher survival for stage IV patients (HR $=0.88,95 \%$ CI [0.71 - 1.09]) nor for patients with metastatic lesions $>5 \mathrm{~cm}(\mathrm{HR}=0.86,95 \%$ CI $[0.69$ - 1.08]) treated with NACT, albeit with some uncertainty due to imprecision. Similarly, no survival benefit was observed in the subgroup of patients with metastatic lesions $>10 \mathrm{~cm}(\mathrm{HR}=0.94,95 \%$ CI $[0.78-1.12])$. NACT was associated with significantly higher rates of complete cytoreduction $(\mathrm{RR}=2.34,95 \%$ CI $[1.48-3.71])$. Severe peri-operative adverse events were less frequent in the NACT arm (RR $=0.34,95 \%$ CI $[0.16-0.72]$. NACT was also associated with a significantly lower risk of post-operative mortality within 28 days $\left(\mathrm{RR}=0.16,95 \%\right.$ CI $[0.06-0.46], \mathrm{I}^{2}=$ $0 \%)$.

Conclusion* Patients with stage III and IV epithelial ovarian cancer undergoing NACT or PDS have similar overall survival. NACT is likely associated with higher rates of complete cytoreduction and lower risk of severe adverse events and perioperative death. 